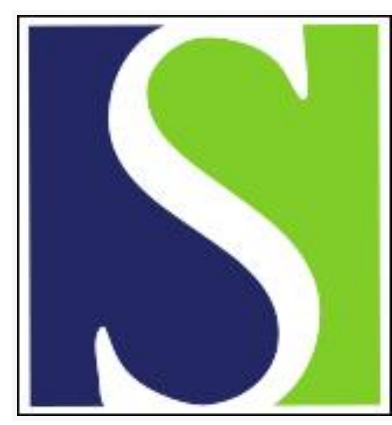

Scand J Work Environ Health 1994;20(4):309-311

https://doi.org/10.5271/sjweh.1392

Issue date: 01 Aug 1994

\section{New epidemics in occupational health.}

by Hernberg $\mathrm{S}$

Affiliation: Finnish Institute of Occupational Health, Helsinki.

This article in PubMed: www.ncbi.nlm.nih.gov/pubmed/7801078 


\section{New epidemics in occupational health}

In May of 1994 the Finnish Institute of Occupational Health arranged an international conference on new epidemics in occupational health. The meeting took place in Helsinki's new Marina Congress Center, tastefully remodeled from an old warehouse and beautifully situated on the waterfront in the heart of the city. The conference attracted 173 participants from 34 countries. The main topics were new musculoskeletal disorders, disorders related to mental stress, new hypersensitivity epidemics, new infectious epidemics, new toxic epidemics, new epidemics caused by physical agents, and research and preventive strategies for new epidemics. Each topic was built around a core of invited keynote lectures. Altogether 20 such presentations were given. They were supplemented by 39 free oral communications, 24 posters, and 6 workshops. The organizers had strongly emphasized the keynote lectures by selecting preeminent scientists to present them. This was indeed a good choice, because many of these lectures represented, in terms of both science and performance, the best one can expect at any conference. Some of the workshops were also interesting, for example, those on sudden deaths, work with visual display terminals, and reproductive hazards, but in general they did not reach quite the same level as the keynote addresses. I was especially disappointed by the workshop on occupational cancer hazards.

All of the keynote addresses cannot be reviewed because of space limitations, but some can be highlighted. In his introductory lecture, Professor Jorma Rantanen, Helsinki, analyzed the concept of epidemics and discussed the criteria for an epidemic. He asked, for example, how "silent" epidemics can be detected and explored. He suggested that the threshold for reporting interesting cases should be lowered and that careful documentation of sporadic observations and suspected cases should be encouraged. Multifactorial and multi-outcome epidemics offer a challenge to researchers, and he recommended that more effective "unofficial" connections be established between researchers and that even data from small-scale studies be made public in order to lower the detection level of new epidemics. He also warned that the tightening data confidentiality legislation, together with the decentralization of data systems and the growing mobility of the work force, will create more problems in the future for the epidemiologist interested in revealing new health risks.

Dr Neil Pearce, Wellington, addressed the problems of research in clusters and the difficulties in interpreting the results. Even a statistically significant result can be misleading. If all similar workplaces are considered (but not studied), the likelihood that an observed cluster is "real" is low, because chance events or some concomitant nonoccupational cause may explain why an excess was observed in one instance. Similar studies in other workplaces would perhaps be "negative," if carried out. If enough workplaces are considered, clusters may occur in one or two of them by pure chance. He said that it is highly likely that at least one workplace, if there are many, will experience an excess of a rare type of cancer every few years or so. Dr Pearce concluded that cluster analyses, although historically very often the first suggestion of a carcinogenic hazard, should be considered as hypothesis-generating only, always requiring confirmation from another study base.

Professor Christer Hogstedt, Stockholm, gave a very interesting keynote address entitled "From Sentinel Observations to Practical Actions." He, in turn, was prepared to give weight to clusters in order to help the early detection of new hazards. He also stressed that regulation succeeds better if the risks are documented in one's "own" country. The involvement of workers also helps early preventive action. Hazard identification research should be closely linked to public health practice, and the surveillance of old risks is warranted. Risk assessment and risk management are important procedures for implementing preventive actions. He finally stressed that, in order to protect the health of citizens, the ethical rule "the benefit of doubt" should be applied when potential hazards are evaluated.

Professor David Wegman, Lowell, Massachusetts, pleaded for the use of well-standardized questionnaires as tools for epidemiologic etiologic research. He listed nine examples in which workers' reports of symptoms had provided the first indication that there was a new toxic epidemic. Although epidemiologists should make more use of systematic symptom reporting, Professor Wegman pointed out that the problem is that symptoms are complex subjective experiences that vary by cultural background, past experience, personality, the meaning of the situation, emotion, and a host of other factors. However, if great care is taken to standardize the questions, high correlations can be obtained between symptoms and measures of exposure, as illustrated by several of the studies he cited. He also pointed out the importance of a good case definition for symptom-based measures of disease and used Schilling's classical byssinosis studies as an example. In competent hands, symptom-based studies can reveal 
early evidence of abnormality, and they often represent independent information. Moreover, symptoms themselves can be the abnormality whenever objective measures do not exist, as, for example, in the case of job burnout. Wegman also discussed the conceptual difference between "acute," "chronic," and "recurrent" symptoms, as well as the measure of "severity." A detailed understanding of symptoms might play an important role in the recognition of new toxic epidemics. He concluded that "a better use of symptom information is not the only way to address the challenges of identifying new epidemics, but a focus on symptoms has the advantage of directing attention to the type of experience that leads individual workers to seek medical attention in the first place."

Dr Barbara Silverstein, Washington, District of Columbia, reviewed the new epidemics of musculoskeletal disorders. She said that this group now represents $61 \%$ of all newly reported workplace disorders in the United States. Although low-back pain is still by far the most prevalent and costly disorder, the incidence rate for upper-limb disorders has increased from 5 per 10000 worker-years in 1981 to almost 30 per 10000 worker-years in 1991. Many studies have shown a strong work-relatedness for hand and wrist symptoms. For example, a survey of seven epidemiologic studies on carpal tunnel syndrome showed work-related odds ratios ranging from 5 to 15 , and another compilation of tendon-related disorders of the hand and wrist revealed work-related odds ratios ranging from 5 to 25 . These figures have to be viewed against the fact that many of these disorders, for example, the carpal tunnel syndrome, are not compensated as occupational diseases in many countries. She concluded that there is ample evidence of an increase both in the reporting and in the true incidence of musculoskeletal disorders and that there is strong evidence for the work-relatedness of these multifactorial disorders.

The topic of the keynote address given by Professor Mats Hagberg, Stockholm, was the pathomechanisms of work-related musculoskeletal disorders. He gave a general model according to which exposure is external to humans and determined by work requirements. The exposure causes an internal dose that is modified by individual capacity and results in a physiological response. A peak load may cause an internal force that results in a response of tissue failure. After having reviewed the pathomechanisms of the most important musculoskeletal disorders, he concluded that individual susceptibility may result in a lower than normal threshold for an exposure resulting in a musculoskeletal disorder. This increased susceptibility can be caused by a disease, a genetic code, or lack of fitness.

Professor Bengt Knave, Stockholm, reviewed some current issues of possible health effects caused by electric and magnetic fields. Although epidemiologic studies on childhood leukemia and residential exposure from power lines seem to indicate a slight increase in risk and although excess leukemia and brain tumors have been reported among workers in "electrical" occupations, there are still unclarities preventing firm conclusions. Some epidemiologic studies have shown adverse pregnancy outcomes and childhood cancer resulting from both maternal and paternal exposure to magnetic fields, but other research groups have not been able to replicate positive results. Epidemiologic studies on pregnancy outcome among operators of video display terminals have mostly been negative, and animal teratogenic studies with similar fields have been too contradictory for conclusions. Some neurobehavioral reactions, such as the slowing of heart rate and electroencephalographic changes after exposure to weak electric or magnetic fields, have been reported. "Hypersensitivity to electricity" has been a hot issue in Sweden, but this ill-defined syndrome seems to be multifactorial in origin, and it is not clear whether electric or magnetic fields are involved at all.

Dr Stephen Olenchock, Morgantown, West Virginia, gave an overview of new hypersensitivity epidemics caused by organic dusts. Organic dusts are especially prevalent in agriculture. There are many different allergens, among them microbial organisms and their toxins, animal danders, insect and rodent parts, and plant materials. Raising animals indoors in confinement units exposes workers to microorganisms of fecal origin, toxins, and danders. Composting is another activity entailing exposure, and the most recent example is the use of gram-negative bacteria to enhance snow-making operations.

Professor Christina Maslach, Berkeley, California, gave a review of job burnout. She defined burnout as a "type of prolonged response to chronic emotional and interpersonal stressors on the job." In terms of outcomes, she said that burnout can lead to a deterioration in the quality of care or service provided to clients or patients. It appears to be a factor in job turnover, absenteeism, and low morale and seems to be correlated with various self-reported indices of personal dysfunction, including physical exhaustion, insomnia, increased use of alcohol and drugs, and marital and family problems. Burnout seems to be specific to the job situation. There is a growing tendency to view burnout as a dynamic process, rather than as a static state.

Professor Marianne Frankenhaeuser, Stockholm, addressed psychosocial factors and occupational health. She stressed that knowledge about human needs, abilities, and constraints provides guidelines for shaping the psychosocial work environment so as to reduce work stress and improve occupational health.

Space does not permit me to add a report of the many interesting workshops, the free communications, the poster sessions, or even all the keynote addresses. Those interested in more details can order the proceedings of the conference, which will be available in October 1994 at a price of FIM 180.00 
from the Publication Office, Finnish Institute of Occupational Health, Topeliuksenkatu 41 a A, FIN00250 Helsinki, Finland.

\section{Sven Hernberg, MD}

Finnish Institute of Occupational Health

Topeliuksenkatu 41 a A

FIN-00250 Helsinki

Finland 